\title{
La Date de Composition de la Bible des sept Estaz du monde de Geufroi de Paris
}

\author{
Julia C. Szirmai ${ }^{1}$ (D \\ Accepted: 10 April 2021 / Published online: 23 April 2021 \\ (c) The Author(s) 2021
}

\begin{abstract}
Among the biblical poems edited by the Équipe de Leyde (founded by Jean-Robert Smeets in 1962), the only text that remained to be studied was the Bible des sept Estaz $d u$ monde by Geufroi de Paris. An extensive project, for the text comprises nearly 22.000 verses. Until now, the researchers who examined Geufroi's Bible have accepted the composition date of 1243 provided by the manuscript on fol. $187 \mathrm{r}^{\circ} \mathrm{b}$, where, in the epilogue of his text, Geufroi asserts to have written his Bible at that date. To support that claim, they based their research mainly on the texts that Geufroi used for the composition of his New Testament, especially the narration of the life of Mary (from the Conception till the Assumption). However, the study of the sources for the Bible's Old Testament (of which we are actually preparing the edition) leads us to another conclusion.
\end{abstract}

Keywords Bible des sept Estaz du monde · Bible translations · Gossuin de Metz · Geufroi de Paris

\section{Les poèmes bibliques en ancien français}

En 1962 Jean-Robert Smeets (1916-2003) fonda l'Équipe de Leyde dans l'intention d'éditer un corpus de traductions-adaptations en vers de la Bible en ancien français. Au cours des années ont paru, dans le cadre du travail de l'Équipe, les textes suivants:

La Chevalerie de Judas Macchabee (Smeets, 1955), la Bible de Macé de La Charité (van der Krabben, 1964; Smeets, 1967 et 1982; Prangsma-Hajenius, 1970; Verhuyck, 1977; Lops, 1982; Smeets \& Mok, 1986), Li Romanz de Dieu et de sa Mere d'Herman de Valenciennes, la Bible de Jehan Malkaraume, la Bible anonyme du Ms. BnF fr. 763 (Szirmai, 1985), La Chevalerie de Judas Macchabee de Gautier

Julia C. Szirmai

J.C.Szirmai@planet.nl

1 Leiden University, Leiden, Netherlands 
de Belleperche (et de Pieros du Riés) (Smeets, 1992) et la Genèse d'Evrat (Boers, 2002).

Depuis 2001, nous avons continué le travail de l'Équipe. ${ }^{1}$ Parmi les 'Bibles intégrales', seule la Bible des .vij. estaz du monde de Geufroi de Paris restait à éditer. Des parties de cette Bible ont été publiées au cours des années: ainsi le Prologue qui contient une Table des matières détaillée (Meyer, 1909), La Passion des Jongleurs. Texte établi d'après la Bible des sept estaz du monde de Geufroi de Paris (Amari Perry, 1981) et le Purgatoire de saint Patrice (White-le Goff, 2019). ${ }^{2}$ Le texte de Geufroi n'a jamais été édité intégralement. Nous en préparons actuellement l'édition.

\section{La Bible des sept Estaz du monde de Geufroi de Paris}

La Bible de Geufroi est une compilation de presque 22.000 vers en octosyllabes à rime plate (Smeets, GRLMA VI/1, 1968). Le texte entier, contenu dans le seul Ms. Paris BnF fr. 1526, contient, à part le Prologue (fol. $1 \mathrm{r}^{\mathrm{o}} \mathrm{a}-1 \mathrm{r}^{\circ} \mathrm{b}$ ) et une Table de matières (fol. 1-8), les sept livres suivants:

Ancien Testament (fol. 9-30)

Nouveau Testament (fol. 30-143)

la Descente en enfer de saint Paul (fol. 144-53)

le Purgatoire (fol. 154-69)

la Condition humaine (fol. 170-79)

Le Temps de l'Antéchrist (fol. 179-82)

la Fin du monde (fol. 183-87).

Nous savons très peu de l'auteur, qui se fait connaître dans le Prologue de sa Bible: Geffroi de Paris, sanz celee,/A ceste Bible compillee/Ces.vij. livres coumence l'on/ Par les.vij. lestres de son non (vv. 15-18). Les spécialistes sont d'avis que «[...] le compilateur était visiblement un homme peu éclairé, incapable, selon toute apparence, de recourir aux sources latines ou même d'apprécier la valeur des écrits en langue vulgaire qu'il ajustait à la suite les uns des autres» (Smeets, GRLMA VI/2, 1970).

L'Ancien Testament de Geufroi rend la Genèse, en mettant l'accent sur la création du monde, ${ }^{3}$ sur la chute d'Adam et Ėve et en élaborant l'histoire de Joseph. Geufroi continue sa translation en racontant l'histoire de Moïse et du peuple d'Israel dans le désert (Exode). Il ne relate, et brièvement, qu'un seul épisode des Nombres:

\footnotetext{
${ }^{1}$ Parmi nos publications, citons dans ce cadre l'édition d'Un Fragment de la Genèse en vers (Szirmai, 2005), et une analyse des Anglo-Norman Bible Stories «The Anglo-Norman Bible Stories in Ms Brit. Libr. Harley 2253», Neophilologus 103, 2 (2019, pp. 161-70).

${ }^{2}$ Voir aussi les publications et les éditions partielles mentionnées par J. R. Smeets dans GRLMA VI/2 (1970, p. 84).

${ }^{3}$ Le début de l'Ancien Testament est tronqué (il manque un feuillet après le fol. $8 \mathrm{r}^{\circ} \mathrm{b}$ ). Manquent également l'histoire de Noé et le début de l'histoire d'Abraham (post fol. $13 \mathrm{v}^{\circ} \mathrm{b}$ ).
} 
après l'épisode du Serpent d'airain, et une partie de la Légende de la Croix qui raconte comment Moïse trouve le bois sacré, Geufroi passe directement aux livres des Rois, en mettant l'accent sur la bataille de David et Goliath, et termine son récit avec le premier jugement et la mort de Salomon. Geufroi n'a pas rendu le Lévitique (matière pas très passionnante en effet), ni le Deutéronome.

L'auteur, dont la langue semble suggérer une provenance de l'Ile-de-France, ${ }^{4}$ avoue être compilateur. Nous avons pu constater que, pour ce qui est de l'Ancien Testament, il puise dans un grand nombre de sources. À part la Vulgate, il met à profit des écrits apocryphes (Vita Adae et Evae (ed. Murdoch \& Tasioulas, 2002), Livre des Secrets d'Énoch ${ }^{5}$ ), Li Romanz de Dieu et de sa Mere d'Herman de Valenciennes (ed. Spiele, 1975), la Queste del saint Graal (ed. Pauphilet, 1980), la Légende de la Croix (Post peccatum Adae (ed. Quinn, 1980)) et l'Image du monde de Gossuin de Metz (ed. Fant, 1886; ed. Prior, 1913).

\section{Historique de la datation}

$\mathrm{Au}$ fol. $187 \mathrm{r}^{\circ} \mathrm{b}$, dans l'épilogue, Geufroi nous fait savoir la date de composition de son poème:

Ci define, ce m'est avis,

La Bible Geffroi de Paris.

Ouez en quel tens fu trestie(e)

Ceste estoire qu'avez oïe:

L'incarnation entendez,

Mil.et. cc. anz touz nombrez

Et.xl.iij. touz de fi.

En cele annee que je di

Fu ceste Bible compilee,

De ces.vij. livres ordenee.

Jean Bonnard (1884, p. 42), Paul Meyer (1909, pp. 257-58), Jean-Robert Smeets (GRLMA VI/2, 1970, p. 84), Anne Jourdan Amari Perry (1981, pp. 95-96), le DLF (1992, p. 183) et Myriam White-Le Goff (2019, p. 627) acceptent cette date de 1243. ${ }^{6}$ À part l'attestation dans le texte même, la datation de la compilation de Geufroi a été basée, jusqu'à maintenant, sur les sources que l'auteur a utilisées pour son Nouveau Testament, comme La Conception Notre Dame de Wace.

\footnotetext{
${ }^{4}$ Bonnard affirme: «La langue n'a pas de caractère dialectal particulier, c'est celle de l'île-de-France». (1884, p. 42); Meyer en donne quelques détails (1909, p. 322). Amari Perry fournit les caractéristiques les plus marquantes dans son édition de la Passion des Jongleurs (1981, pp. 97-104).

${ }^{5}$ Robert H. Charles (Ed.). (1913), The Apocrypha and Pseudepigrapha of the Old Testament, vol. II, pp. 425ss. Cf. Quinn (1980, p. 143).

${ }^{6}$ White-Le Goff date le texte de «avant 1243», sans préciser pourquoi elle croit à une date antérieure à celle donnée par Geufroi.
} 
Ce sont surtout les emprunts à Li Regres Nostre Dame de Huon de Cambrai (dans les livres II et V de Geufroi) qui ont fait éclater une discussion entre Långfors (1907, pp. CXXXVIII-CXLII), éditeur du poème, et Meyer (1909, pp. 257-58). En 1901, Gröber (Grundriss II, 1, pp. 759 et 837) affirme que la Parabole des faux amis qui fait partie du Regres est postérieure à 1244. Selon lui, la strophe 230 contient une allusion à la conquête de Jérusalem, fin 1244 (il s'agit d'une plainte sur Jérusalem où les Turcs détiennent le Saint Sépulcre). Långfors se range du côté de Gröber, mais ajoute que d'autres strophes du Regres font référence à la septième Croisade de saint Louis, ce qui mènerait à une datation d'entre 1244 et 1248 . Une objection à cette hypothèse, affirme-t-il, est le fait que Geufroi de Paris a inséré de nombreux passages du Regres dans sa Bible que le compilateur date de 1243. Or, Geufroi n'a pas pu connaître ce poème en 1243. Comme l'affirme Långfors (1907, p. CXXXVIII, n.1): «Je dirai un peu plus loin que, pour la composition de la Bible des.VII. estaz $d u$ monde, la date de 1243 ne me paraît pas certaine.» À la page CXLI il fait remarquer: «[...] je serais donc porté à ne pas considérer comme certaine la date de 1243 qui doit désigner l'année où la Bible des.VII. estaz du monde a été composée» (Långfors, 1907).

Långfors propose de corriger la date en 1263 (p. CXLII, n.2), mais son hypothèse est contestée par Meyer (1909, pp. 257-58), qui croit que les allusions dans $\mathrm{Li}$ Regres ne réfèrent pas à la croisade de saint Louis, mais à la prise de Jérusalem en 1187.

\section{Nouvelles perspectives}

Långfors fait remarquer (p. CXLII, n.2): «Au point de vue paléographique, une erreur entre 1243 et 1263 serait très simple». Il propose donc de corriger.xl.iij. en.lx. iij. Une telle correction, pourtant, demande une intervention dans le texte qui est non seulement bien forte, mais aussi quelque peu arbitraire, la septième croisade étant terminée en 1254. Le déplacement d'une simple lettre n'est pas justifié du point de vue du contenu.

Aux hypothèses de Långfors et de Meyer, nous ajouterions une troisième: sans devoir corriger le texte, on pourrait lire.xl.vj. au lieu de.xl.iii. et dater le texte de 1246. Contrairement au nombre.vij. (ligne 10), les lettres qui devraient représenter.iij. ne portent pas de traits au-dessus (ligne $5 \mathrm{du}$ fragment suivant).

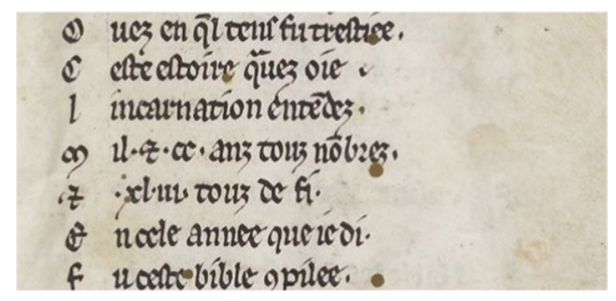


Mil.et. cc. anz touz nombrez/Et .xl.vj. touz de fi

Gallica, Paris, BnF fr. 1526 , fol. $187 \mathrm{r}^{\circ} \mathrm{b}$

De plus, on n'a qu'à regarder l'écriture de $v$ intérieur (par exemple au lignes 6-7 du fol. $187 \mathrm{r}^{\circ} \mathrm{a}$ : servistes) pour voir que ce qu'on a lu comme .iij. peut bien représenter $v j$.

\section{a wiangtes arpuatite. o ue wus lettutes.}

Aus angres de perversitélQue vous servistes

Gallica, Paris, BnF fr. 1526 , fol. $187 \mathrm{r}^{\circ} \mathrm{a}$

À part les considérations paléographiques, le contenu du premier livre de la Bible de Geufroi peut fournir un autre argument pour étayer notre hypothèse. Nous avons constaté que ceux qui se sont occupés des sources de la Bible se sont surtout concentrés sur les textes que Geufroi a utilisés pour son Nouveau Testament. Ils n'ont guère considéré les sources de l'Ancien Testament, sauf pour relever quelques rapports avec Li Romanz de Dieu et de sa Mere d'Herman de Valenciennes et avec certaines légendes, dont la Légende de la Croix. Or, avant de commencer le récit d'Adam et Ėve, Geufroi présente une brève description du paradis terrestre (tronquée du début), qui est suivie d'un chapitre sur l'enfer et la naissance des quatre vents. Ces vers montrent des rapports frappants avec l'Image du Monde de Gossuin de Metz. Il existe de ce texte trois rédactions en vers et une en prose (Fant, 1886; Prior, 1913; Sentili, 2005).

Nous ferons suivre le fragment, en vers, de la première rédaction, du texte correspondant de Gossuin, en signalant en italiques les correspondances avec le texte de Geufroi.

\begin{tabular}{lll}
\hline Geufroi vv. 874-901 & Ms. BnF fr. 2173 (fol. 16r $\left.\mathrm{r}^{\circ} \mathrm{b}-35 \mathrm{r}^{\circ} \mathrm{b}\right)$ & \\
& $\begin{array}{l}\text { Por ce que la terre est pesanz } \\
\text { Plus que nus des.iiij. elemenz }\end{array}$ & fol. $16 r^{\circ} \mathrm{b}$ \\
& {$[\ldots]$} & \\
Rubr & Rubr & \\
Ci parole d'enfer et des entrailles/ & Ou enfer siet et en quel leu & fol. $30 r^{\circ} a$ \\
de la terre et des.iiij. venz & & \\
\hline
\end{tabular}

Enmi la terre, et u plus bas,

Fist Diex enfer, je n'en dout pas

Ilec est li parfonz abismes,

Einsi l'establi li Hautismes,

Pour ce qu'il est le plus pesant

De touz les autres ellemenz

Damediex li Peres ceu leu

Fist touz plains de souffre et de feu
Cil leus qui en terre est enfers

Je di que c'est li drois enfers

[...]

Car trop est laiz. vilz et oscurs

Et plus pesant que nule rien

Par coi em puet entendre bien

Que ou plus bas leu a fait sont estre

[...] 
Et c'est ou mileu de la terre

fol. $30 r^{\circ} b$

Touz jorz art et toz jors ardra,

Ore oez et si ne vos griés

Tant com li siecles durera

Comment enfers ou mileu siet

Cil leus qui est en terre enfers

De la terre (...)

Est apellez li droiz enfers

[...]

C'est li puiz d'enfer et le gouffre

Tout ausint a dedens la terre

Qui est plains de feu et de soufre,

.I. lieu qui abismes a non

fol. $30 v^{o} a$

D'oribleté et de douleur,

[...]

De chaut, de froit et de pueur,

De touz les maus qu'en puet penser

Qui pu(e)ent nuire ne grever

Itant vos di de celui leu

Qu'il est plains de seufre et de feu

Hideus, puant et plains d'ordure

[...]

En terre fist maint autre leu

Qui sont plain de souffre et de feu

Que plus art plus longuement dure

[...]

Dedenz terre, en cavez grans,

La enclost Diex les.iiij. vans

En terre a maint autre leus

fol. $31 r^{\circ} a$

Qui sont orrible et perilleus

Que il fet par le mont aler

Et amont et aval soufler,

De feu et de froideur orible

[...]

Si que par le deboutement

fol. $32 v^{o} b$

Quant $i$ l espart et tonne et pluet

Es nues font l'eaue geler,

En nois et en gresles muer

Des ondes naissent aucuns vent

En cavernes qui sont soz terre

[...]

Les grans greles qu'en estre vienent

fol. $34 \mathrm{r}^{\mathrm{o} a}$

Par icele manière $[\ldots]$

Quant en l'air vienent aucun vent

Dont froidures issent souvent

De la moisteur qu'en l'air est nee

fol. $34 v^{o} b$

De la terre trait agelee

[...]

Car espars ne tonnoires grant

N'estfors deboutements de vent

Qui s'encontrent desor les nues

[...]

Issi vienent souvantes foiz

fol. $35 r^{\circ} b$

Nues, pluies, venz etescroiz ${ }^{7}$

Gossuin de Metz et Geufroi de Paris donnent une description de l'enfer qui contient les données suivantes: Dieu créa l'enfer au milieu (c'est-à-dire, au plus bas) de la terre. C'est l'abîme profond. L'enfer est le plus lourd de tous les éléments. Le lieu est plein de souffre et de feu qui brûle éternellement. Un lieu rempli de froideur, de

\footnotetext{
7 Notre transcription. Var. ms BnF fr. 2021: gresles et noiz. Selon Prior (1913, pp. 8-9), la version en prose a été composée probablement en 1246, mais en tout cas avant la seconde rédaction en vers. Le texte présente les mêmes éléments que les versions en vers.
} 
chaleur, de douleur et de choses horribles. Il y a encore d'autres lieux pareils sur la terre.

Cette description est suivie d'une explication des phénomènes naturels causés par les vents. Dieu a enfermé dans des cavernes profondes les quatre vents qui soufflent dans le monde et qui causent la tonnerre, la foudre et le grêle. Car la tonnerre n'est autre chose que les vents qui se heurtent dans les nues.

Il est intéressant de noter que, selon Prior, «Le chapitre sur l'enfer semble être l'ouvrage de Gossuin lui-même sans emploi direct de sources. Honorius Aug. (I, 37) lui a peut-être servi de base». ${ }^{8}$ Comment, sinon par le texte de Gossuin, Geufroi a-til pu se procurer ces détails? ${ }^{9}$

\section{Conclusion}

Il ne fait pas de doute que Geufroi a puisé dans une version du texte de Gossuin pour les vers 874 jusqu' au 901 inclus. Il s'en suit que sa Bible ne peut avoir été composée en 1243, puisque la première rédaction en vers de l'Image du Monde date de $1246,{ }^{10}$ la deuxième et la troisième «des années suivantes» (Cf. Sentili, 2005, chap. III).

Depuis 1884, lorsque Bonnard a fait connaître la Bible de Geufroi (Bonnard, 1884), on a accepté la date de 1243, que le compilateur lui-même semble indiquer dans son texte. À la base de l'étude des sources de l'Ancien Testament de Geufroi, pourtant, la date de 1246 semble s'imposer.

Les observations les plus récentes de M. Gloria Ríos Guardiola $\left(2011\right.$, p. 17) ${ }^{11}$ et de M. Boulton (2015, pp. 58, 59, 67 et 121) concernant la date de Li Regres Nostre Dame ne semblent pas démentir cette conclusion.

En résumé, nous pouvons avancer trois arguments pour dater la Bible de Geufroi de Paris de 1246: du point de vue paléographique rien ne s'oppose à la lecture 1246: Mil.et. cc. anz [...] Et.xl.vj. [...]. Les correspondances, souvent textuelles, entre le texte de Geufroi et celui de Gossuin de Metz témoignent d'un rapport de contenu indéniable entre la Bible et l'Image du Monde, texte qui doit être daté de 1246 et qui, de plus, semble être le propre de Gossuin.

\footnotetext{
${ }^{8}$ Prior (1913, p. 139, n. B). Prior réfère à l'Imago Mundi d'Honorius Augustodunensis.

9 Voir note 3.

10 Nous remercions Chantal Connochie-Bourgne de cette précision (dans quelques manuscrits apparaît la date de 1245) et de ses informations additionnelles. Voir son article «Nature» et «clergie» dans l'œuvre de vulgarisation scientifique de Gossuin de Metz (Image du Monde 1245) (1994, pp. 9-27, n.1). Fant (1886) note que les copistes des nombreux manuscrits qui existent de l'Image du Monde hésitent entre les dates, qui varient de 1245 à 1247.

11 «Li Regrès Nostre Dame habría sido escrita algunes años antes de la primera cruzada de S. Luis dirigada contra Egipto y Siria (1248-1254) (Gallet 1964: 389) y antes de 1250 según Paul Zumthor (1954: 273). Ciertas alusiones prueban que es posterior a la toma de Jerusalem por parte de los Sarracenos en 1244; sin embargo, numeros versos de este poema se encuentran en la Bible des septs états du monde de Geufroi de Paris, datada en 1243 (Langlois 1909, p. 32)».
} 
Open Access This article is licensed under a Creative Commons Attribution 4.0 International License, which permits use, sharing, adaptation, distribution and reproduction in any medium or format, as long as you give appropriate credit to the original author(s) and the source, provide a link to the Creative Commons licence, and indicate if changes were made. The images or other third party material in this article are included in the article's Creative Commons licence, unless indicated otherwise in a credit line to the material. If material is not included in the article's Creative Commons licence and your intended use is not permitted by statutory regulation or exceeds the permitted use, you will need to obtain permission directly from the copyright holder. To view a copy of this licence, visit http://creativecommons.org/licen ses/by/4.0/.

\section{Références}

Amari Perry, A. J. (Ed.). (1981). La Passion des Jongleurs. Texte établi d'après la Bible des sept estaz du monde de Geufroi de Paris. Beauchesne.

Boers, W. (Ed.). (2002). La Genèse d'Evrat. Ver Luisant.

Bonnard, J. (1884). La Bible française au Moyen Age. Imprimerie nationale.

Boulton, M. (2015). Sacred fictions of medieval France. Narrative theology in the Lives of Christ and the Virgin, 1150-1500. Brewer.

Charles, R. H. (Ed.). (1913). The Apocrypha and Pseudepigrapha of the Old Testament. Clarendon Press.

Connochie-Bourgne, C. (1994). «Nature» et «clergie» dans l'œvre de vulgarisation scientifique de Gossuin de Metz (Image du Monde 1245). Comprendre et maîtriser la nature au Moyen Age: mélanges offerts à Guy Beaujouan (Droz/Champion), pp. 9-27.

Fant, C. (Ed.). (1886). L'Image du monde. Poème inédit du milieu du XIIIe siècle: étudié dans ses divers rédactions françaises d'après les manuscrits des bibliothèques de Paris et de Stockholm. EDV Berling.

Gröber, G. (1901). Grundriss der romanischen Philologie, II, I. Trübner.

Hasenohr, G., \& Zink, M. (Eds.). (1992). Dictionnaire des Lettres françaises. Fayard.

Långfors, A. (Ed.). (1907). Huon le roi de Cambrai, Li Regres Nostre Dame, publié d'après tous les manuscrits connus. Champion.

Lops, R. (Ed.). (1982). La Bible de Macé de La Charité. Brill/Universitaire Pers.

Meyer, P. (1909). Notice sur la Bible des sept estaz du monde de Geufroi de Paris. Notices et extraits de la Bibliothèque Nationale et d'autres bibliothèques, vol. XXXIX, pp. 255-322.

Murdoch, B., \& Tasioulas, J. A. (Eds.). (2002). The apocryphal Lives of Adam and Eve. University of Exeter Press.

Pauphilet, A. (Ed.). (1980). La Queste del saint Graal. Champion.

Prangsma-Hajenius, A. M. I. (Ed.). (1970). La Bible de Macé de La Charité III. Universitaire Pers.

Prior, O. H. (Ed.). (1913). L'Image du monde de maître Gossuin. Payot.

Quinn, E. C. (1980). The penitence of Adam: A study of the Andrius Ms. (Bibliothèque Nationale Fr. 95, folios 380r-394v). Romance Monographs 36. University of Mississippi.

Rios Guardiola, M. G. (Ed.). (2011). Huon de Cambrai. Edicions de la Universidad de Murcia.

Sentili, S. (2005). La tradition manuscrite de l'Image du monde. Fortune et diffusion d'une encyclopédie du XIII ${ }^{e}$ siècle. Diplôme d'archiviste paléographe, École des chartes, Paris (theses.enc.sorbonne.fr/2005/ sentili).

Smeets, J. R. (1968). Les traductions, adaptations et paraphrases de la Bible en vers. GRLMA VI/1, pp. 48-57, GRLMA VI/2 (1970).

Smeets, J. R. (Ed.). (1955). La Chevalerie de Judas Macchabee. Thèse Groningen.

Smeets, J. R. (Ed.). (1967). La Bible de Macé de La Charité, I. Universitaire Pers.

Smeets, J. R. (Ed.). (1982). La Bible de Macé de La Charité V. Brill/Universitaire Pers.

Smeets, J. R. (Ed.). (1992). La Chevalerie de Judas Macchabee. Van Gorcum.

Smeets, J. R., \& Mok, Q. I. M. (Eds.). (1986). La Bible de Macé de La Charité. Universitaire Pers.

Spiele, I. (Ed.). (1975). Li Romanz de Dieu et de sa Mere d'Herman de Valenciennes. Presse universitaire de Leyde.

Szirmai, J. C. (Ed.). (1985). La Bible anonyme du Ms. Paris B.N.f. fr. 763. Édition critique. Rodopi.

Szirmai, J. C. (Ed.). (2005). Un Fragment de la Genèse en vers (fin XIII ${ }^{e}$-début XIV siècle), éd. du Ms. Brit. Libr. Harley 3775. Droz. 
Szirmai, J. C. (2019). The Anglo-Norman Bible Stories in Ms Brit. Libr. Harley 2253. Neophilologus 103, 2 , pp. 161-70.

van der Krabben, H. C. M. (Ed.). (1964). La Bible de Macé de La Charité IV. Brill/Universitaire Pers.

Verhuyck, P. E. R. (Ed.). (1977). La Bible de Macé de La Charité II. Universitaire Pers.

White-le Goff, M. (Ed.). (2019). Marie de France, Le Purgatoire de saint Patrick accompagné des autres versions françaises en vers et du Tractatus de Purgatorio sancti Patricii de H. de Saltrey. Champion.

Publisher's Note Springer Nature remains neutral with regard to jurisdictional claims in published maps and institutional affiliations. 